\title{
Dimethyl Fumarate Attenuates Oxaliplatin-Induced Peripheral Neuropathy without Affecting the Anti-tumor Activity of Oxaliplatin in Rodents
}

\author{
Anna Miyagi, ${ }^{\#}$ Takehiro Kawashiri,,\# Shiori Shimizu, Nao Shigematsu, Daisuke Kobayashi, and \\ Takao Shimazoe \\ Department of Clinical Pharmacy and Pharmaceutical Care, Graduate School of Pharmaceutical Sciences, Kyushu \\ University; 3-1-1 Maidashi, Higashi-ku, Fukuoka 812-8582, Japan. \\ Received November 2, 2018; accepted December 25, 2018
}

\begin{abstract}
Oxaliplatin has been used as a first choice for colorectal, gastric and pancreatic cancer, but it induces peripheral neuropathies. Dimethyl fumarate (DMF) is an oral drug for multiple sclerosis with neuroprotective effects on oxidative stress. Using both in vivo and in vitro models, we investigated the effects of DMF on oxaliplatin-induced peripheral neuropathy and other side effects, as well as on the anti-tumor activity of oxaliplatin. Repeated intraperitoneal injection of $4 \mathrm{mg} / \mathrm{kg}$ oxaliplatin (twice per week for 4 weeks) caused mechanical allodynia (as revealed by the von Frey tests), cold hyperalgesia (as revealed by the acetone tests), and axonal degeneration in the sciatic nerve of rats. Co-administration of oral DMF $(200 \mathrm{mg} / \mathrm{kg}$, five times per week for 4 weeks) relieved oxaliplatin-induced mechanical allodynia but not cold hyperalgesia, and ameliorated axonal degeneration. In addition, DMF did not exacerbate oxaliplatin-induced body weight loss or bone marrow suppression, such as reduction in red blood cells, white blood cells, neutrophils and lymphocytes. Furthermore, DMF did not inhibit the anti-tumor activity of oxaliplatin in any cultured cancer cell line (C26, mouse colon carcinoma; HCT116, human colon carcinoma; MKN45, human gastric adenocarcinoma; MIA PaCa-2, human pancreatic carcinoma) or C26-bearing mice. These results suggest that DMF prevents oxaliplatin-induced mechanical allodynia and axonal degeneration without affecting the anti-tumor activity of oxaliplatin. Therefore, DMF may be useful for managing oxaliplatin-induced chronic peripheral neuropathy.
\end{abstract}

Key words dimethyl fumarate; oxaliplatin; peripheral neuropathy; mechanical allodynia; axonal degeneration

\section{INTRODUCTION}

Oxaliplatin has been used as a first choice for treating colorectal, gastric and pancreatic cancer. However, it frequently induces severe side effects, such as acute and chronic peripheral neuropathies. Cold hyperesthesia occurs in the early stages after oxaliplatin administration as the acute neuropathy, but in most cases, it is transient and can get reversed after withdrawing drug. ${ }^{1-3)}$ It is thought that this acute neuropathy is not caused by the morphological alternation of the nerve. ${ }^{4,5}$ As patients undergo repeated treatments, chronic neuropathy or sensory and motor nerve dysfunction occurs. ${ }^{3,6,7)}$ It is a dose-limiting factor during treatment. Therefore, occasionally, not only dose reduction but also oxaliplatin discontinuation is required to revert the chronic neuropathic effects. Thus, peripheral neuropathy is a major clinical issue in oxaliplatinbased chemotherapy.

Many clinical and non-clinical studies on chemotherapyinduced peripheral neuropathy have been conducted. However, in the clinical practice guidelines for chemotherapy-induced neuropathy established by the American Society of Clinical Oncology in 2014, ${ }^{8)}$ there are no drugs recommended for the prevention of neuropathy. Although a detailed mechanism of neuropathy development due to chemotherapy has not been identified, it is reported that oxaliplatin causes morphological degeneration, including axonal disorder, neuronal cell damage

\footnotetext{
${ }^{\#}$ These authors contributed equally to this work.

* To whom correspondence should be addressed. e-mail: tkawa@med.kyushu-u.ac.jp
}

and myelin alternation. ${ }^{9-13)}$ It has recently reported that the knockout of nuclear factor-erythroid-2-related factor 2 (Nrf2) aggravates oxaliplatin-induced mechanical allodynia and that the activation of the Nrf2 signaling pathway ameliorates oxaliplatin-induced neuropathy in mice. ${ }^{14)}$

Dimethyl fumarate (DMF) is a drug for treating multiple sclerosis. It has a neuroprotective effect on oxidative stress via the Nrf2 pathway. ${ }^{15,16)}$ Previously, we reported that DMF and its metabolite monomethyl fumarate (MMF) ameliorate neurite degenerations, which caused by oxaliplatin, cisplatin and bortezomib, via activation of Nrf2 pathway in PC12 cell line. ${ }^{17)}$

In this study, using in vivo models, we investigated whether DMF 1) ameliorates oxaliplatin-induced peripheral neuropathy both behaviorally and morphologically, 2) exacerbates other oxaliplatin-induced systemic toxicities in rats and 3) inhibits the anti-tumor activity of oxaliplatin.

\section{MATERIALS AND METHODS}

Animals Sprague-Dawley rats (6-week-old, male, 200-250 g, Japan SLC, Inc., Shizuoka, Japan) were used for assessing peripheral neuropathy and other systemic toxicities. $\mathrm{BALB} / \mathrm{c}$ mice (6-week-old, male, 15-25g, Japan SLC, Inc.) were used for testing the anti-tumor activity. Animals were housed in groups of four per cage, with lights on from 7:00 to $19: 00 \mathrm{~h}$.

All experiments were approved by the Experimental Ani- 
mal Care and Use Committee of Kyushu University according to the National Institutes of Health guidelines and followed the International Association for the Study of Pain Committee for Research and Ethical Issues guidelines for animal research. $^{18)}$

Cell Culture Murine colon adenocarcinoma C26 cells were obtained from Cell Resource Center for Biomedical Research, Institute of Development, Aging and Cancer, Tohoku University (Miyagi, Japan). Human colon carcinoma HCT116 cells and human gastric carcinoma MKN45 cells were obtained from Riken (Saitama, Japan). Human pancreatic carcinoma MIA PaCa2 cells were a kind gift from Dr. Soichi Takiguchi of the National Kyushu Cancer Center (Fukuoka, Japan). For the cultures of C26, MKN45 and MIA PaCa2 cells, RPMI 1640 medium (Sigma-Aldrich Co. LLC., St. Louis, MO, U.S.A.) supplemented with $2 \mathrm{mM}$ L-glutamine and $10 \%$ fetal bovine serum (FBS) was used. HCT116 cells were cultured in Dulbecco's modified Eagle's medium (Gibco ${ }^{\mathrm{TM}}$; Thermo Fisher Scientific Inc., Waltham, MA, U.S.A.) with $2 \mathrm{mM}$ L-glutamine and 10\% FBS. All cell lines were cultured at $37^{\circ} \mathrm{C}$ in air supplemented with $5 \% \mathrm{CO}_{2}$ under humidified conditions.

Drugs Oxaliplatin (ELPLAT ${ }^{\circledR}$ i.v. infusion solution) was obtained from Yakult Honsha Co., Ltd. (Tokyo, Japan) and was dissolved in 5\% glucose solution. DMF and MMF were purchased from Wako Pure Chemical Industries, Ltd. (Osaka, Japan) and was dissolved in $0.5 \%$ methyl cellulose or dimethyl sulfoxide for use in in vivo and in vitro studies. In the oxaliplatin-induced peripheral neuropathy model, oxaliplatin $(4 \mathrm{mg} / \mathrm{kg})$ or vehicle ( $5 \%$ glucose solution) was injected intraperitoneally (i.p.), twice per week for 4 weeks (on days 1, 2, 8, 9, 15, 16, 22 and 23). DMF (100 and $200 \mathrm{mg} / \mathrm{kg}$ ) or vehicle $(0.5 \%$ methyl cellulose) was administered orally ( $p e r$ os (p.o.)), five times per week for 4 weeks (on days 1-5, 8-12, 15-19 and 22-26). All drugs were administered at a volume of $1 \mathrm{~mL} / \mathrm{kg}$. The drug doses were determined based on a previous report. ${ }^{19)}$

Assessment for Mechanical Allodynia (von Frey Test) The effect of DMF on mechanical allodynia was assessed using the von Frey test. The method of von Frey test was described in previous report. ${ }^{20)}$ This test was performed on each of the following days: before the first drug administration (day 0 ) and on days 4, 8, 11, 15, 18, 22, 25 and 29. Each rat was placed in a wire mesh box for $30 \mathrm{~min}$ before testing for the adaptation. Von Frey filaments (Aesthesio ${ }^{\circledR}$; DanMic Global, LLC, San Jose, CA, U.S.A.) were applied to the mid-plantar skin of each hind paw for $6 \mathrm{~s}$, and the withdrawal threshold was determined by the modified up-down method.

Assessment for Cold Hyperalgesia (Acetone Test) The acetone test was performed on day 26 for assessment of cold hyperalgesia. The method was described in previous report. ${ }^{19)}$ Rats were adapted to the wire mesh box in the same manner as von Frey test. Acetone (Hayashi Pure Chemical Ind., Ltd.) was sprayed onto the plantar skin, and the number of withdrawal responses was counted for $40 \mathrm{~s}$.

Assessment of Sciatic Axonal Degeneration Rat sciatic nerves were harvested after the anesthesia by sevoflurane (Wako Pure Chemical Industries, Ltd.) on day 30. After the fix in $2 \%(\mathrm{w} / \mathrm{v})$ glutaraldehyde followed by $8 \%(\mathrm{w} / \mathrm{v})$ sucrose substitution, nerves were embedded in Epon and sliced and then stained with toluidine blue. ${ }^{20)}$ The sections were evalu- ated by light microscopy (CKX41SF; Olympus Co., Tokyo, Japan). The fiber diameter, the axon circularity, the myelin thickness, the g-ratio (the ratio of axonal diameter to fiber diameter), and the number of axon in three animals from each group were calculated. ${ }^{21)}$

Assessment for Systemic Toxicities Body weight loss and blood cell counts were assessed for determining systemic toxicities. Body weights were measured on days $0,4,8,11,15$, $18,22,25$ and 29. On day 30, blood samples were taken from rat tail vein, and the total blood cell counts were measured using a Thoma haemocytometer (Erma, Inc., Tokyo, Japan). Subsequently, Giemsa staining was performed to evaluate the percentage of white blood cells (neutrophils/lymphocytes) in the total blood count, and the number of each type of blood cell (red blood cells, white blood cells, neutrophils and lymphocytes) was counted.

Tumor Cytotoxicity Assay Cell lines C26, HCT116, MKN45 and MIA PaCa-2 were seeded at a density of $2 \times 10^{4}$ cells/well onto 24-well plates (Thermo Fisher Scientific Inc.)

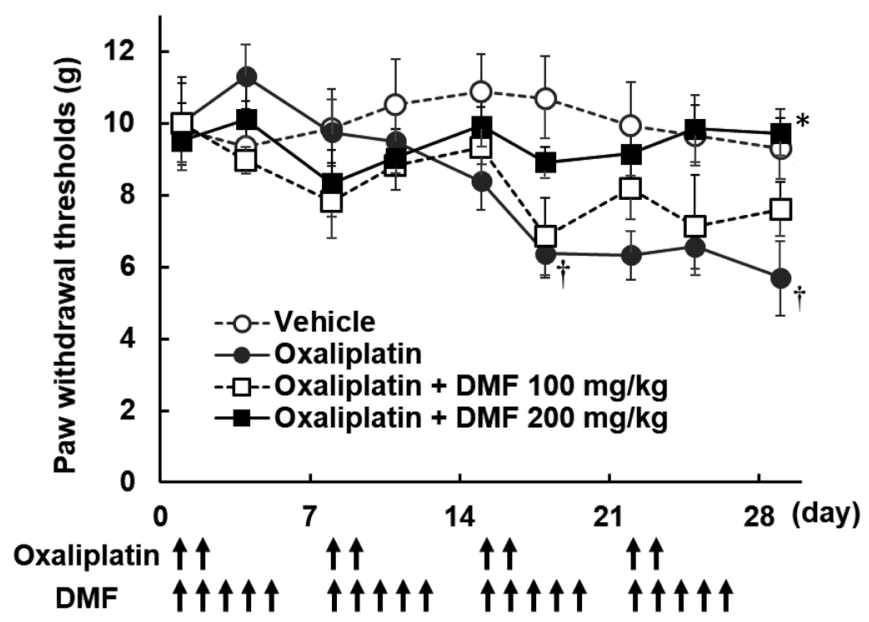

Fig. 1. Effects of Dimethyl Fumarate (DMF) on Oxaliplatin-Induced Mechanical Allodynia in Rats

Oxaliplatin $(4 \mathrm{mg} / \mathrm{kg})$ was administered i.p. twice per week for 4 weeks. DMF $(100$ or $200 \mathrm{mg} / \mathrm{kg})$ was administered p.o. five times per week for 4 weeks. The von Frey test was performed before the first drug administration (on day 0 ) and on days $4,8,11,15,18,22,25$ and 29 . Values are expressed as the mean \pm S.E.M. of six animals. On days 18 and 29, oxaliplatin group showed significant lower thresholds compared with vehicle $\left({ }^{\dagger} p<0.05\right)$. On day 29 , oxaliplatin + DMF $200 \mathrm{mg} / \mathrm{kg}$ group showed a significant higher threshold compared with oxaliplatin-alone treatment $(* p<0.05)$.

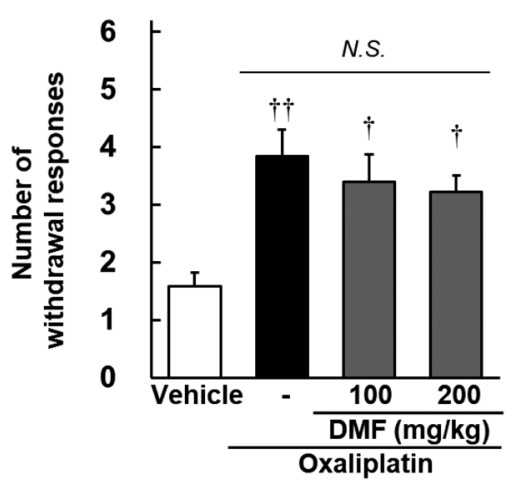

Fig. 2. Effects of DMF on Oxaliplatin-Induced Cold Hyperalgesia in Rats

The acetone test was performed on day 26. Values are expressed as the mean \pm S.E.M. of six animals. ${ }^{\dagger} p<0.05$ and ${ }^{\dagger} p<0.01$ compared with vehicle treatment. 
and were used for experiments on the following day. Cells were exposed to oxaliplatin $(10 \mu \mathrm{M})$ and $\operatorname{DMF}(0,0.3,1,3$, or $10 \mu \mathrm{M})$ or its metabolite $\operatorname{MMF}(0,0.3,1,3$, or $10 \mu \mathrm{M})$ for $24 \mathrm{~h}$. The cell viability was assessed by WST- 8 method (Cell Counting Kit-8; Dojindo Laboratory, Kumamoto, Japan).

Tumor Growth Analysis Using Mouse Model C-26 cells $\left(1.5 \times 10^{6}\right.$ cells $)$ were subcutaneously implanted in the right paw of each mouse. Three days after the tumor implantation, the drug administrations were started. Oxaliplatin $(6 \mathrm{mg} / \mathrm{kg}$, i.p.) was administered on days $1,2,8$ and 9 , and
DMF $(300 \mathrm{mg} / \mathrm{kg}$, p.o.) was administered on days $1-5$ and $8-12$. The dose of oxaliplatin was adjusted higher than the dose for rats because $4 \mathrm{mg} / \mathrm{kg}$ (twice a week) of oxaliplatin could not attenuate the tumor growth in a preliminary experiment using mice. The oxaliplatin doses were as per the protocols from previous reports. ${ }^{13,22)}$ The dose of DMF was also increased at the same ratio as oxaliplatin. The tumor volumes were measured on days $4,6,8,11,13$ and 15 and were calculated as follows: Volume $\left(\mathrm{mm}^{3}\right)=\pi / 6 \times$ thickness $(\mathrm{mm}) \times$ length $(\mathrm{mm}) \times$ width $(\mathrm{mm})$.

\section{A)}

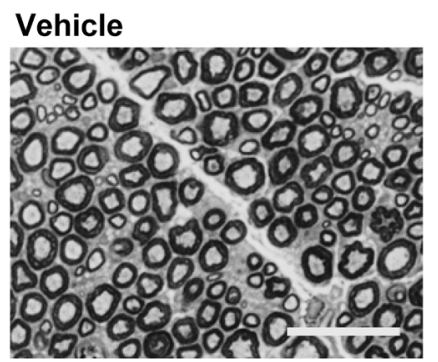

Bar: $50 \mu \mathrm{m}$

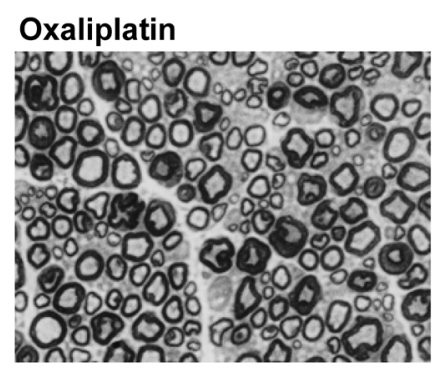

Oxaliplatin + DMF

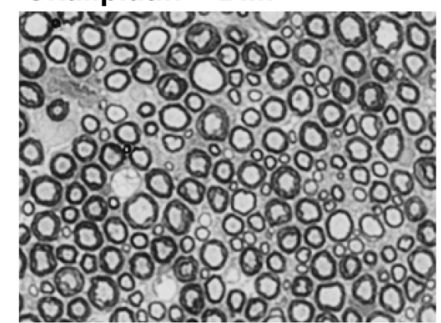

B) Fiber diameter

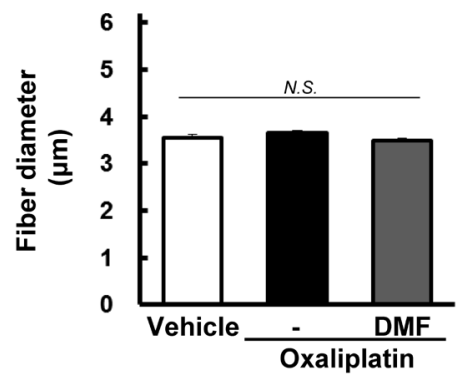

D) Myelin thickness

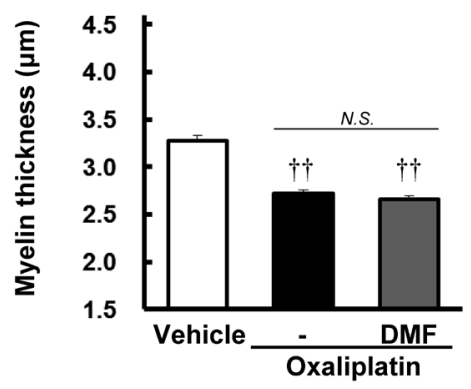

F) Number of axons

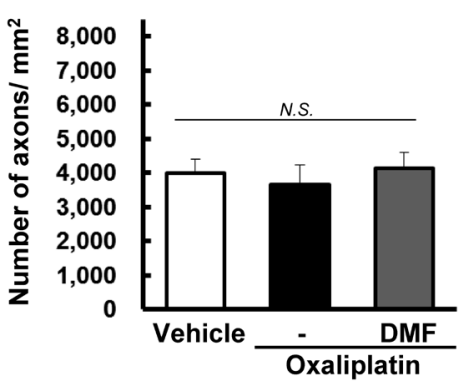

\section{C) Axon circularity}

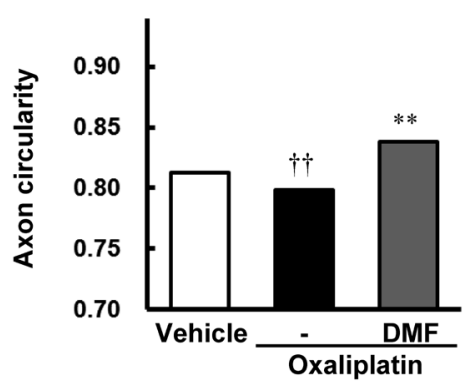

E) g-ratio

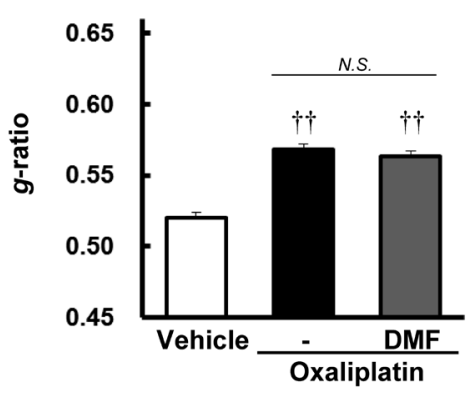

Fig. 3. Effects of DMF on Oxaliplatin-Induced Axonal Degeneration in the Sciatic Nerves of Rats

The sciatic nerves were harvested, and samples were stained with toluidine blue on day 30 . Photographs (A) were originally magnified $40 \times$. Scale bar $=50 \mu \mathrm{m}$. Fiber diameter (B), axon circularity (C), myelin thickness (D), g-ratio (E), and number of axons (F) were analyzed. Values are expressed as the mean \pm S.E.M. of three animals. ${ }^{+1} p<0.01$ compared with vehicle, $* * p<0.01$ compared with oxaliplatin alone. 
Statistical Analysis The data are shown as the mean \pm standard error of the mean (S.E.M.). Statistical analysis was performed using one-way ANOVA, followed by the Tukey-Kramer test (Statview; Abacus Concepts, Berkley, CA, U.S.A.) to determine the differences between the groups. A probability level of $p<0.05$ was accepted as statistically significant.

\section{RESULTS}

Effects of DMF on Mechanical Allodynia in Oxaliplatin-Treated Rats Compared with vehicle treatment, oxaliplatin treatment $(4 \mathrm{mg} / \mathrm{kg}$, i.p., twice per week) significantly decreased the paw withdrawal thresholds on days 18 and 29 $(p<0.05$; Fig. 1). Co-administration of DMF at a dose of $200 \mathrm{mg} / \mathrm{kg}$ (p.o., five times a week) significantly alleviated the oxaliplatin-induced reduction in the paw withdrawal thresholds on day 29 ( $p<0.05$; Fig. 1). However, co-administration of DMF at a dose of $100 \mathrm{mg} / \mathrm{kg}$ showed no such effect (Fig. 1).

Effects of DMF on Cold Hyperalgesia in OxaliplatinTreated Rats The acetone test was performed to assess the effects of DMF on cold hyperalgesia on day 26. Compared with vehicle treatment, oxaliplatin treatment $(4 \mathrm{mg} / \mathrm{kg}$, i.p.) significantly increased the number of withdrawal responses $(p<0.01$; Fig. 2). Co-administration of DMF $(100$ and $200 \mathrm{mg} / \mathrm{kg}$ ) showed no effect on the oxaliplatin-induced increase in the number of withdrawal responses (Fig. 2).

Effects of DMF on the Oxaliplatin-Induced Histological Change in the Sciatic Nerves of Rats Oxaliplatin treatment $(4 \mathrm{mg} / \mathrm{kg}$, i.p.) induced the degeneration of myelinated fibers in the sciatic nerves of rats (Fig. 3A), and co-administration of DMF ameliorated this degeneration (Fig. 3A). The axon circularity, a quantitative index of degeneration, was significantly decreased in oxaliplatin-treated rats compared with that in the vehicle-treated rats $(p<0.01$; Fig. 3C). However, co-administration of DMF significantly prevented the oxaliplatin-induced reduction in the axon circularity $(p<0.01$; Fig. $3 \mathrm{C})$. The myelin sheaths of oxaliplatin group were significant thinner than vehicle group $(p<0.01$; Fig. 3D). Moreover, the $g$-ratios in oxaliplatin-treated rats were higher than vehicle $(p<0.01$; Fig.
$3 \mathrm{E})$. However, these morphological changes were not ameliorated by DMF administrations $(p<0.01$; Figs. 3D, E). The fiber diameter and the number of axons were not changed by oxaliplatin treatments (Figs. 3B, F).

Effects of DMF on Oxaliplatin Toxicity During the course of the experiment, no rat in any group showed abnormal behavior or died. Compared with vehicle treatment, oxaliplatin treatment significantly lowered the body weight after day 11 (Fig. 4A). No significant difference was observed in the body weight between the oxaliplatin-only group and the DMF co-administration group at any time (Fig. 4A). However, compared with rats treated with the vehicle, those treated with oxaliplatin alone showed significant lower levels of red blood cells ( $p<0.01$; Fig. 4B), white blood cells $(p<0.01$; Fig. 4 C), neutrophils $(p<0.01$; Fig. 4D) and lymphocytes $(p<0.01$; Fig. $4 \mathrm{E})$. Co-administration of DMF $(200 \mathrm{mg} / \mathrm{kg})$ did not exacerbate the decrease in any blood cell count (Figs. 4B-E).

Effects of DMF or MMF on the Oxaliplatin-Induced Tumor Cytotoxicity in Cultured Cell Lines In cultured C26, HCT116, MKN45 and MIA PaCa2 cells, the exposure to oxaliplatin $(10 \mu \mathrm{M})$ significantly inhibited cell growth $(p<0.01)$. DMF $(0.3-10 \mu \mathrm{M})$ or its metabolite MMF $(0.3-10 \mu \mathrm{M})$ showed no effect on the oxaliplatin-induced cytotoxicity (Figs. $5 \mathrm{~A}-\mathrm{H})$.

Effects of DMF on the Anti-tumor Activity of Oxaliplatin in Tumor Cell-Implanted Mice Oxaliplatin treatment $(6 \mathrm{mg} / \mathrm{kg}$, i.p.) significantly inhibited the increase of tumor volumes compared with the vehicle treatment $(p<0.01)$. DMF $(300 \mathrm{mg} / \mathrm{kg}$, p.o.) showed no effect on the oxaliplatin-induced inhibition of tumor growth (Fig. 6).

\section{DISCUSSION}

In this study, oxaliplatin treatment caused mechanical allodynia and cold hyperalgesia, which is consistent with previously reported observations. ${ }^{19)}$ Co-administration of $200 \mathrm{mg} / \mathrm{kg}$ DMF prevented the occurrence of oxaliplatininduced mechanical allodynia. Thus, DMF may be useful for preventing chronic peripheral neuropathy associated with oxaliplatin chemotherapy. However, DMF had no such effect

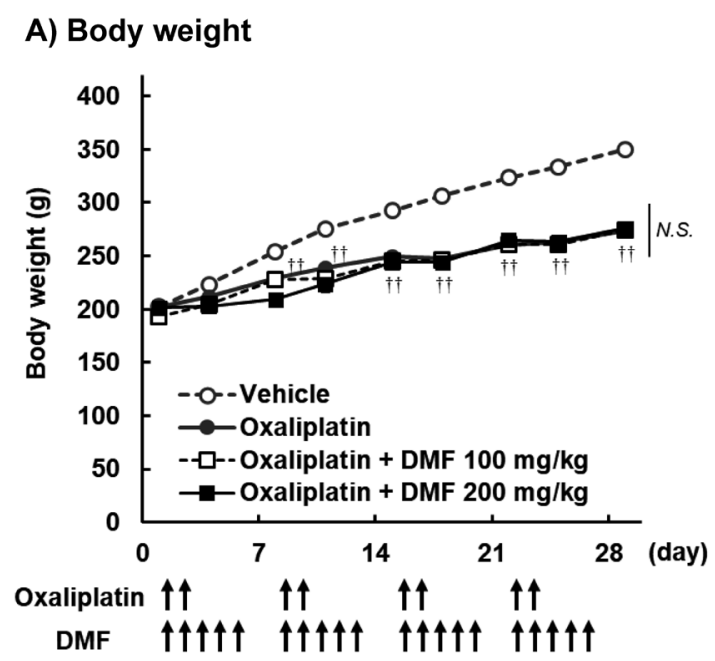

B) Red blood cell

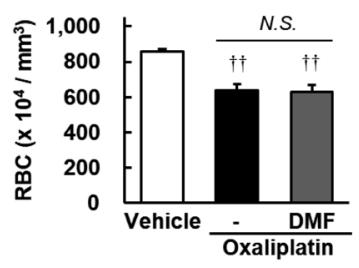

D) Neutrophil

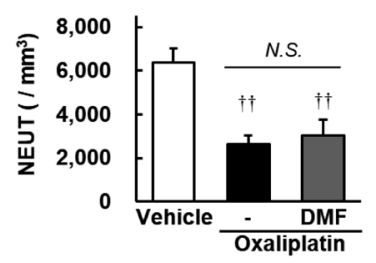

C) White blood cell

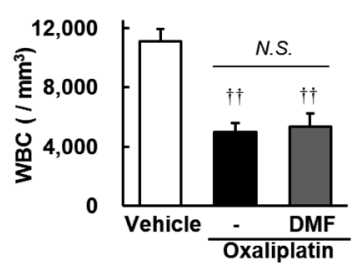

E) Lymphocyte

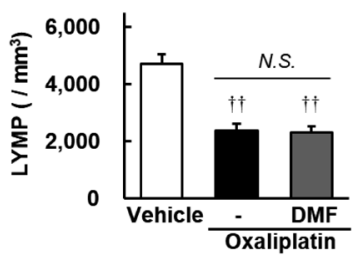

Fig. 4. Effects of DMF on Oxaliplatin-Induced Weight Loss and Myelosuppression in Rats

Body weight was measured on days $0,4,8,11,15,18,22,25$ and 29 (A). On day 30, blood samples were taken from rat tail vein, and the total blood count was measured using a Thoma haemocytometer. Subsequently, Giemsa staining was performed to calculate the counts of each type of blood cells: red blood cells (B), white blood cells (C), neutrophils (D) and lymphocytes (E). Data are shown as the mean \pm S.E.M. of six animals. ${ }^{\dagger} p<0.01$ compared with vehicle. 


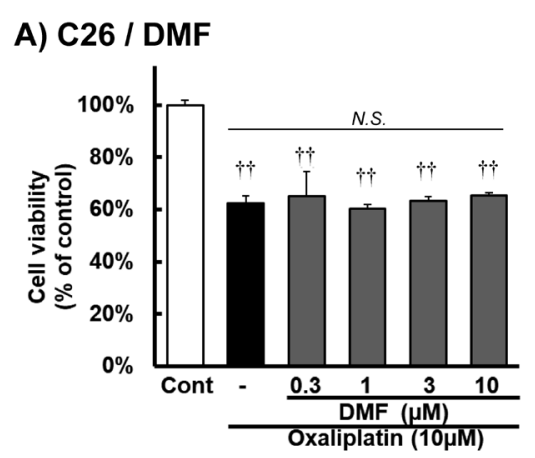

C) HCT116 / DMF

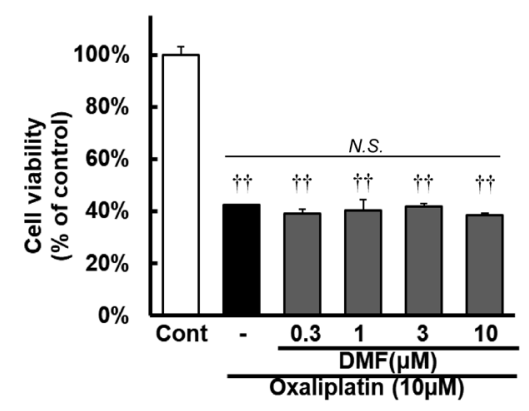

E) MKN45 / DMF

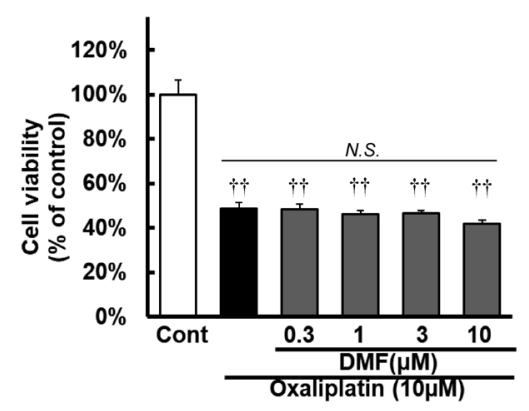

G) MIA PaCa2 / DMF

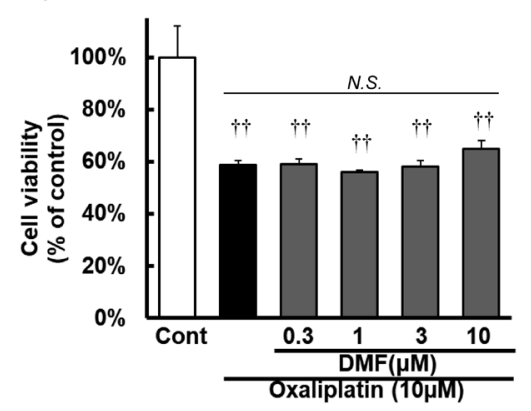

B) C26 / MMF

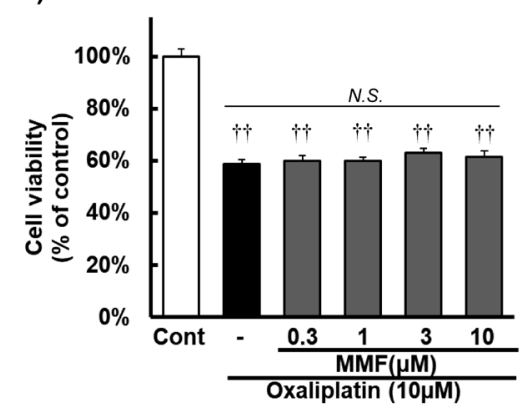

D) HCT116 / MMF

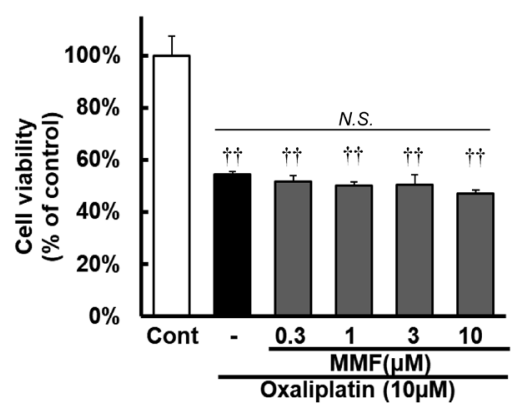

F) MKN45 / MMF

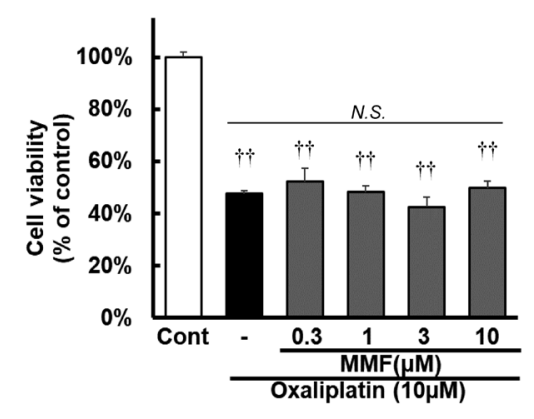

H) MIA PaCa2 / MMF

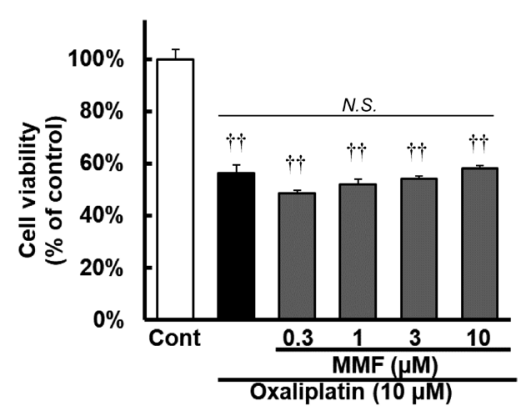

Fig. 5. Effects of DMF or Its Metabolite Monomethyl Fumarate (MMF) on Oxaliplatin-Induced Tumor Cytotoxicity in Cultured Cell Lines

C26 (A, B), HCT116 (C, D), MKN45 (E, F) and MIA PaCa-2 (G, H) cells were incubated with oxaliplatin (10 $\mu$ M) for $24 \mathrm{~h}$ in the presence or absence of various concentrations $(0.3-10 \mu \mathrm{M})$ of DMF (A, C, E and G) or MMF (B, D, F and H). Cell viability was measured by WST-8 assay. Values are expressed as the mean \pm S.E.M. $(n=4-8) .{ }^{\dagger} p<0.01$ compared with control.

on cold hyperalgesia.

Histological investigation on day 30 showed that the oxaliplatin treatment induced axonal degeneration of myelinated fibers in the sciatic nerve, indicating axonopathy, and that co-administration of DMF prevented this degeneration. Neurodegeneration, such as neuronopathy, axonopathy and myelinopathy, are involved in chronic neuropathy development associated with oxaliplatin treatment. ${ }^{23-26)}$ In addition, a correlation between the repair of axonal degeneration and the amelioration of mechanical allodynia has previously been reported. ${ }^{13,20)}$ However, there is reportedly no association between acute neuropathy and neurodegeneration. ${ }^{4,5,19,27)}$ And some studies indicated the transient receptor potential channels (TRPA1 and TRPM8) are involved in oxaliplatin-induced acute cold hyperalgesia. ${ }^{27,28)}$ These may be the reason why no effect of the co-administration of DMF on oxaliplatin-induced 


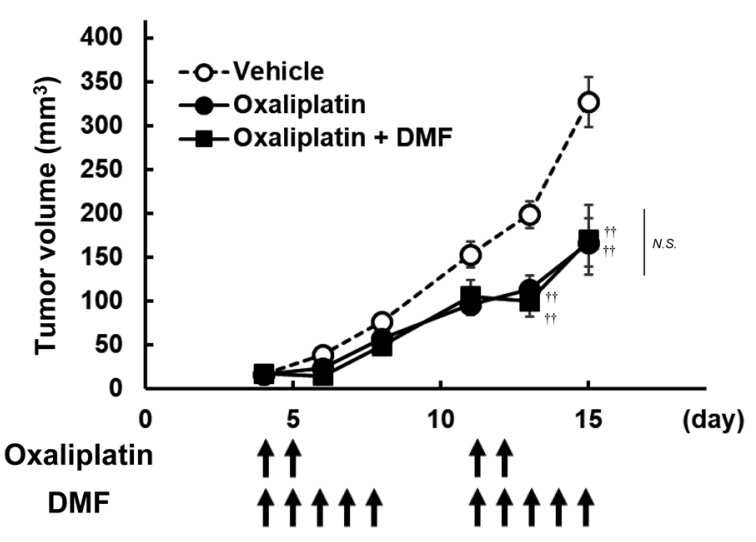

Fig. 6. Effects of DMF on the Anti-tumor Activity of Oxaliplatin in Tumor Cell-Implanted Mice

C26 cell-implanted mice were treated with oxaliplatin $(6 \mathrm{mg} / \mathrm{kg}$, i.p. $)$ twice a week and DMF $(300 \mathrm{mg} / \mathrm{kg}$, p.o.) five times per week for 2 weeks. The tumor volumes were calculated as follows: Volume $(\mathrm{mm} 3)=\pi / 6 \times$ thickness $(\mathrm{mm}) \times$ length $(\mathrm{mm}) \times$ width $(\mathrm{mm})$. Values are expressed as the mean \pm S.E.M. $(n=8-11)$ ${ }^{+1} p<0.01$ compared with vehicle.

cold hyperalgesia was observed in the acetone test. In this study, oxaliplatin induced not only the decrease in the axon circularity but also the decrease in the myelin thickness and the increase in the $g$-ratio in sciatic nerve sections. Unfortunately, DMF did not ameliorate these myelin disorders. Taken together, the anti-neuropathic effects of DMF were likely due to the amelioration of axonal degeneration, but not myelin disorders.

The neuroprotective effects of DMF are achieved by relieving oxidative stress through the enhancement of the Nrf2 pathway. ${ }^{15,16)} \mathrm{Nrf2}$ activity is normally regulated by keap1, but in response to oxidative stress, Nrf2 becomes free and starts producing antioxidant proteins, such as glutathione reductase, superoxide dismutase and thioredoxin. ${ }^{29)}$ Meanwhile, many previous studies have indicated that oxidative stress is one of the causes of oxaliplatin-induced peripheral neuropathy. ${ }^{30-33)}$ Our previous study indicated that DMF and MMF ameliorate neurite degenerations, which caused by oxaliplatin, cisplatin and bortezomib, via activation of Nrf2 pathway in cultured cell. ${ }^{17)}$ Thus, in this study, it is possible that the co-administration of DMF ameliorated axonopathy and mechanical allodynia by relieving oxidative stress caused by oxaliplatin treatment in the nerve cells.

Although DMF rarely causes serious side effects, some studies have reported that it causes lymphopenia ${ }^{34,35)}$; therefore, we were concerned that DMF may worsen myelosuppression during oxaliplatin treatment. However, our results showed that the co-administration of DMF during oxaliplatin treatment caused no further decrease in any blood cell count and no more weight loss than that caused by oxaliplatin treatment in rats, indicating that the co-treatment using oxaliplatin and DMF has little additional risk.

It was previously reported that excessive spinal transmission activities, such as spinal glutamate uptake, spinal $N$-methyl-Daspartate (NMDA) receptor NR2B subunit overexpression and CaMKII activation, are involved in oxaliplatin-induced chronic neuropathy in rodents. ${ }^{36-38)}$ It is considered that these spinal changes are likely due to peripheral neurodegeneration. In this study, DMF ameliorated not only the symptoms of oxaliplatin-induced peripheral neuropathy but also oxaliplatininduced histological disorders. Thus, DMF may be a suitable drug candidate for managing oxaliplatin-induced peripheral neurotoxicity.

In conclusion, our results suggest that the repeated administration of DMF alleviates oxaliplatin-induced mechanical allodynia and axonal degeneration. In addition, co-administration of DMF does not worsen the side effects of oxaliplatin nor does it inhibit the anti-tumor effects of oxaliplatin. Therefore, DMF may be useful for preventing oxaliplatin-induced peripheral neuropathy.

Acknowledgments This work was supported by JSPS KAKENHI Grant-in-Aid for Young Scientists (B), Grant number 16K19184.

Conflict of Interest The authors declare no conflict of interest.

\section{REFERENCES}

1) Extra JM, Marty M, Brienza S, Misset JL. Pharmacokinetics and safety profile of oxaliplatin. Semin. Oncol., 25 (Suppl. 5), 13-22 (1998).

2) Argyriou AA, Cavaletti G, Briani C, Velasco R, Bruna J, Campagnolo M, Alberti P, Bergamo F, Cortinovis D, Cazzaniga M, Santos C, Papadimitriou K, Kalofonos HP. Clinical pattern and associations of oxaliplatin acute neurotoxicity: a prospective study in 170 patients with colorectal cancer. Cancer, 119, 438-444 (2013).

3) Grothey A. Oxaliplatin-safety profile: neurotoxicity. Semin. Oncol., 30 (Suppl. 15), 5-13 (2003).

4) Wilson RH, Lehky T, Thomas RR, Quinn MG, Floeter MK, Grem JL. Acute oxaliplatin-induced peripheral nerve hyperexcitability. $J$. Clin. Oncol., 20, 1767-1774 (2002).

5) Lehky TJ, Leonard GD, Wilson RH, Grem JL, Floeter MK. Oxaliplatin-induced neurotoxicity: acute hyperexcitability and chronic neuropathy. Muscle Nerve, 29, 387-392 (2004).

6) Starobova H, Vetter I. Pathophysiology of chemotherapy-induced peripheral neuropathy. Front. Mol. Neurosci., 10, 174 (2017).

7) Pasetto LM, D’Andrea MR, Rossi E, Monfardini S. Oxaliplatinrelated neurotoxicity: how and why? Crit. Rev. Oncol. Hematol., 59, 159-168 (2006)

8) Hershman DL, Lacchetti C, Dworkin RH, Lavoie Smith EM, Bleeker J, Cavaletti G, Chauhan C, Gavin P, Lavino A, Lustberg MB, Paice J, Schneider B, Smith ML, Smith T, Terstriep S, Wagner-Johnston N, Bak K, Loprinzi CL, American Society of Clinical Oncology. Prevention and management of chemotherapy-induced peripheral neuropathy in survivors of adult cancers: American Society of Clinical Oncology clinical practice guideline. J. Clin. Oncol., 32, 1941-1967 (2014).

9) Boyette-Davis J, Dougherty PM. Protection against oxaliplatininduced mechanical hyperalgesia and intraepidermal nerve fiber loss by minocycline. Exp. Neurol., 229, 353-357 (2011).

10) Renn CL, Carozzi VA, Rhee P, Gallop D, Dorsey SG, Cavaletti G. Multimodal assessment of painful peripheral neuropathy induced by chronic oxaliplatin-based chemotherapy in mice. Mol. Pain, 7, 29 (2011).

11) Tsutsumi K, Yamashita Y, Ushio S, Kawashiri T, Kaname T, Fujita S, Oishi R, Egashira N. Oxaliplatin induces hypomyelination and reduced neuregulin 1 expression in the rat sciatic nerve. Neurosci. Res., 80, 86-90 (2014).

12) Kono $T$, Suzuki $Y$, Mizuno $K$, Miyagi C, Omiya $Y$, Sekine $H$, Mizuhara Y, Miyano K, Kase Y, Uezono Y. Preventive effect of oral goshajinkigan on chronic oxaliplatin-induced hypoesthesia in rats. Sci. Rep., 5, 16078 (2015).

13) Fujita S, Ushio S, Ozawa N, Masuguchi K, Kawashiri T, Oishi R, Egashira N. Exenatide facilitates recovery from oxaliplatin-induced 
peripheral neuropathy in rats. PLOS ONE, 10, e0141921 (2015).

14) Yang Y, Luo L, Cai X, Fang Y, Wang J, Chen G, Yang J, Zhou Q, Sun X, Cheng X, Yan H, Lu W, Hu C, Cao P. Nrf2 inhibits oxaliplatin-induced peripheral neuropathy via protection of mitochondrial function. Free Radic. Biol. Med., 120, 13-24 (2018).

15) Scannevin RH, Chollate S, Jung MY, Shackett M, Patel H, Bista P, Zeng W, Ryan S, Yamamoto M, Lukashev M, Rhodes KJ. Fumarates promote cytoprotection of central nervous system cells against oxidative stress via the nuclear factor (erythroid-derived 2)-like 2 pathway. J. Pharmacol. Exp. Ther., 341, 274-284 (2012).

16) Al-Jaderi Z, Maghazachi AA. Utilization of dimethyl fumarate and related molecules for treatment of multiple sclerosis, cancer, and other diseases. Front. Immunol., 7, 278 (2016).

17) Kawashiri T, Miyagi A, Shimizu S, Shigematsu N, Kobayashi D, Shimazoe T. Dimethyl fumarate ameliorates chemotherapy agentinduced neurotoxicity in vitro. J. Pharmacol. Sci., 137, 202-211 (2018).

18) Zimmermann M. Ethical guidelines for investigations of experimental pain in conscious animals. Pain, 16, 109-110 (1983).

19) Sakurai M, Egashira N, Kawashiri T, Yano T, Ikesue H, Oishi R. Oxaliplatin-induced neuropathy in the rat: involvement of oxalate in cold hyperalgesia but not mechanical allodynia. Pain, 147, 165-174 (2009).

20) Kawashiri T, Egashira N, Watanabe H, Ikegami Y, Hirakawa S, Mihara Y, Yano T, Ikesue H, Oishi R. Prevention of oxaliplatin-induced mechanical allodynia and neurodegeneration by neurotropin in the rat model. Eur. J. Pain, 15, 344-350 (2011).

21) Yamamoto S, Kawashiri $T$, Higuchi $H$, Tsutsumi K, Ushio S, Kaname T, Shirahama M, Egashira N. Behavioral and pharmacological characteristics of bortezomib-induced peripheral neuropathy in rats. J. Pharmacol. Sci., 129, 43-50 (2015).

22) Ushio S, Egashira N, Sada H, Kawashiri T, Shirahama M, Masuguchi K, Oishi R. Goshajinkigan reduces oxaliplatin-induced peripheral neuropathy without affecting anti-tumour efficacy in rodents. Eur. J. Cancer, 48, 1407-1413 (2012).

23) McKeage MJ, Hsu T, Screnci D, Haddad G, Baguley BC. Nucleolar damage correlates with neurotoxicity induced by different platinum drugs. Br. J. Cancer, 85, 1219-1225 (2001).

24) Cavaletti G, Tredici G, Petruccioli MG, Dondè E, Tredici P, Marmiroli P, Minoia C, Ronchi A, Bayssas M, Etienne GG. Effects of different schedules of oxaliplatin treatment on the peripheral nervous system of the rat. Eur. J. Cancer, 37, 2457-2463 (2001).

25) Jamieson SM, Liu J, Connor B, McKeage MJ. Oxaliplatin causes selective atrophy of a subpopulation of dorsal root ganglion neurons without inducing cell loss. Cancer Chemother. Pharmacol., 56, 391-399 (2005).

26) Al Moundhri MS, Al-Salam S, Al Mahrouqee A, Beegam S, Ali BH. The effect of curcumin on oxaliplatin and cisplatin neurotoxicity in rats: some behavioral, biochemical, and histopathological studies. J. Med. Toxicol., 9, 25-33 (2013).

27) Kawashiri T, Egashira N, Kurobe K, Tsutsumi K, Yamashita Y, Ushio S, Yano T, Oishi R. L type $\mathrm{Ca}^{2+}$ channel blockers prevent oxaliplatin-induced cold hyperalgesia and TRPM8 overexpression in rats. Mol. Pain, 8, 7 (2012)

28) Nakagawa T, Kaneko S. Roles of transient receptor potential ankyrin 1 in oxaliplatin-induced peripheral neuropathy. Biol. Pharm. Bull., 40, 947-953 (2017).

29) Hayes JD, McMahon M. NRF2 and KEAP1 mutations: permanent activation of an adaptive response in cancer. Trends Biochem. Sci., 34, 176-188 (2009).

30) McQuade RM, Carbone SE, Stojanovska V, Rahman A, Gwynne RM, Robinson AM, Goodman CA, Bornstein JC, Nurgali K. Role of oxidative stress in oxaliplatin-induced enteric neuropathy and colonic dysmotility in mice. Br. J. Pharmacol., 173, 3502-3521 (2016).

31) Di Cesare Mannelli L, Zanardelli M, Landini I, Pacini A, Ghelardini C, Mini E, Bencini A, Valtancoli B, Failli P. Effect of the SOD mimetic MnL4 on in vitro and in vivo oxaliplatin toxicity: Possible aid in chemotherapy induced neuropathy. Free Radic. Biol. Med., 93, 67-76 (2016).

32) Di Cesare Mannelli L, Zanardelli M, Failli P, Ghelardini C. Oxaliplatin-induced oxidative stress in nervous system-derived cellular models: could it correlate with in vivo neuropathy? Free Radic. Biol. Med., 61, 143-150 (2013).

33) Di Cesare Mannelli L, Zanardelli M, Failli P, Ghelardini C. Oxaliplatin-induced neuropathy: oxidative stress as pathological mechanism. Protective effect of silibinin. J. Pain, 13, 276-284 (2012).

34) Fox RJ, Chan A, Gold R, Phillips JT, Selmaj K, Chang I, Novas M, Rana J, Marantz JL. Characterizing absolute lymphocyte count profiles in dimethyl fumarate-treated patients with MS: Patient management considerations. Neurol. Clin. Pract., 6, 220-229 (2016).

35) Alroughani R, Ahmed SF, Behbehani R, Al-Hashel J. Effectiveness and safety of dimethyl fumarate treatment in relapsing multiple sclerosis patients: real-world evidence. Neurol. Ther., 6, 189-196 (2017).

36) Yamamoto S, Ushio S, Egashira N, Kawashiri T, Mitsuyasu S, Higuchi H, Ozawa N, Masuguchi K, Ono Y, Masuda S. Excessive spinal glutamate transmission is involved in oxaliplatin-induced mechanical allodynia: a possibility for riluzole as a prophylactic drug. Sci. Rep., 7, 9661 (2017).

37) Mihara Y, Egashira N, Sada H, Kawashiri T, Ushio S, Yano T, Ikesue H, Oishi R. Involvement of spinal NR2B-containing NMDA receptors in oxaliplatin-induced mechanical allodynia in rats. $\mathrm{Mol}$. Pain, 7, 8 (2011).

38) Shirahama M, Ushio S, Egashira N, Yamamoto S, Sada H, Masuguchi K, Kawashiri T, Oishi R. Inhibition of $\mathrm{Ca}^{2+} /$ calmodulindependent protein kinase II reverses oxaliplatin-induced mechanical allodynia in rats. Mol. Pain, 8, 26 (2012). 